\title{
Stress contributions in colloidal suspensions: the smooth, the rough and the hairy
}

\author{
Bram Schroyen, Chiao-Peng Hsu, Lucio Isa, Peter Van Puyvelde, Jan Vermant
}

October 5, 2018

Even the most simple colloidal suspensions, consisting of spherical particles in a Newtonian matrix, display very rich and nonlinear properties. For example the rheological properties vary over orders of magnitudes and in the same suspension shear thinning and shear thickening of the viscosity can occur when the stress is varied. These phenomena arise because of a complex interplay between the colloidal interactions and the hydrodynamic ones. Much has been learned by looking at the simplest case of Brownian hard sphere suspensions and mapping colloidal particles onto these as effective hard spheres [1,2]. Recently however, there has been an interest in more complicated colloidal systems and how properties such as discontinuous shear thickening and dilatancy are influenced $[3,4]$. Specifically, the role of surface roughness and friction has been the subject of several recent studies [5-9]. In some systems friction is even invoked as the sole controlling factor of the rheological behaviour, but it is not clear why for example hydrodynamic forces can now be neglected $[4,7]$. the choice for the word 'now' seems strange

Several methods exist to interrogate colloidal forces and are suited to investigate the role of surface roughness or softness as well. Single particle measurements, using for example colloidal probe AFM or optical tweezers, enable a direct and quantitative measurement of surface topography and the static interaction potentials [10-13]. Although highly useful, such single particle level experiments remain challenging and may not always yield statistical information. Moreover, it is not always possible to investigate the intriguing interplay between hydrodynamic effects and surface characteristics. Other approaches have been to look at collective properties. Varying the volume fraction is useful as it changes the interparticle spacing in a known and averaged manner. For instance the osmotic pressure [14] or linear viscoelastic properties as a function of volume fraction have been used to derive the colloidal pair potential [15-19]. More recently the rate dependent flow curve of colloidal dispersions has been fitted and particle level friction coefficients are inferred from the rheological curves [20,21]. Many, if not all of these approaches focus on single aspects and do not enable one to interrogate the fascinating interplay between hydrodynamic and colloidal forces and how these are modulated by surface topography.

snap niet 'guided by statistical theories'

In this letter, high frequency linear rheology is used to evaluate specifically and directly this interplay, averaged over the suspension volume. In particular we use the volume fraction and frequency dependency of the viscous and elastic moduli to understand if and how hydrodynamic forces are altered for rough and soft particles, guided by statistical theories [22-24]. Conceptually, the total deviatoric stress tensor in a suspension $\boldsymbol{\Sigma}$ can be written as [22, 24]:

$$
\boldsymbol{\Sigma}=2 \eta \dot{\boldsymbol{\epsilon}}+\boldsymbol{\Sigma}_{p}=2 \eta \dot{\boldsymbol{\epsilon}}+\left(\boldsymbol{\Sigma}^{H}+\boldsymbol{\Sigma}^{B}+\boldsymbol{\Sigma}^{P}\right) .
$$


Here, $\dot{\boldsymbol{\epsilon}}$ is the bulk rate of strain tensor so that $2 \eta \dot{\boldsymbol{\epsilon}}$ represents the stress in the suspending fluid. The particle contribution to the stress $\boldsymbol{\Sigma}_{p}$ can be further decomposed into direct hydrodynamic $\left(\boldsymbol{\Sigma}^{H}\right)$, Brownian $\left(\boldsymbol{\Sigma}^{B}\right)$ and interparticle $\left(\boldsymbol{\Sigma}^{P}\right)$ contributions. The hydrodynamic terms are responsible for a high-frequency (HF) limiting viscosity.

To investigate what happens to $\boldsymbol{\Sigma}_{p}$ for topographically complex particles, we experiment at frequencies that are high compared to the relaxation frequencies associated with Brownian motion in the suspensions. For these type of measurements, two conditions need to be met. First, sufficiently sensitive high frequency measurements (not accessible by commercial instruments) of both the viscous and the elastic moduli are needed. High frequency implies being faster than the relaxation time, which for Brownian systems is given by $\tau_{p, B}[16,22,25]$ :

$$
\tau_{p, B}=\frac{a^{2}}{\mathcal{D}_{s s}}=\frac{6 \pi \eta_{\infty}^{\prime} a^{3}}{k_{B} T}=\frac{1}{2 \pi f_{p, B}}
$$

with $k_{B} T$ the thermal energy scale and $a$ the particle size. Eq. 2 also sets the length scale under investigation, since $\tau_{p, B}$ describes the time that a particle requires to travel a distance similar to its own size. A homebuilt piezo shear rheometer was used to probe the linear stress response over an extended frequency range $(f=0.001-2000$ $\mathrm{Hz}$ ) (suppl. mat. S2) [26]. We measure $\eta_{\infty}^{\prime}$ experimentally (figs. S.4) as well as the particle size and can identify an effective $\mathrm{HF}$ regime.

voldoende duidelijk dat first en second samenhoren nog?

Second, we use model soft and rough systems. PMMA particles stabilised by means of densely grafted PHSA chains are used as soft sterically stabilised systems. A better understanding of the stabiliser synthesis led to a controllable brush chain length [27] and we use systems with a varying number of PHSA repeat units $(3.8 \rightarrow 5.5 \rightarrow 46$, as determined from NMR, suppl. mat. S1.1) and core size (tab. S.1 and fig. S.1). Controlled roughness is obtained by employing charge-stabilised raspberrylike silica colloids with tuneable asperities [13,28] (suppl. mat. S1.2). The surface was varied from smooth (silica-SM) to increasingly rough (silica-RO.4 $\rightarrow$ silica-RO.5) (tab. S.2 and fig. S.2). The suspending medium was generally chosen to a) minimise van der Waals forces and b) optimise the viscosity for increased signal strength for $\mathrm{HF}$ rheometry and to shift the Brownian relaxation times to lower frequencies.

Defining the volume fraction for suspensions with varying surface topography is not trivial. Here, we propose an effective hydrodynamic volume fraction $\phi_{\text {eff }}$ derived from the limiting $\mathrm{HF}$ viscosity $\eta_{\infty}^{\prime}$ (fig. S.6, suppl. mat. S4) $[29,30]$. The resulting $\phi_{\text {eff }}$ relates directly to the hydrodynamic stresses induced by the particles, and is hence more suited as a basis for comparing the stress contributions. ook hier

interferentie hebt met dispersiekwaliteit

The linear viscoelastic moduli reveal the typical behaviour of a suspension with Brownian motion (figs. S.4-S.5, suppl. mat. S3). To have a more clearcut representation of the particle level stresses we rescale both axes. The frequency in the experiments is rescaled by the theoretical limit for the high frequency regime such that $\alpha^{p, B}=f \cdot \frac{2 \pi a^{2}}{\mathcal{D}_{s s}}>1$ for comparing different volume fractions, or alternatively from the experimentally observed $\alpha^{*}$ above which the high frequency regime was reached when comparing the systems with vastly different topographies. The linear viscoelastic moduli are rescaled to remove bulk hydrodynamic stresses $\left(\eta_{\infty}^{\prime} \cdot 2 \pi f\right)$ and are further normalised by this value. The resulting data give the normalised extra stresses present on top of the hydrodynamic ones. These stresses increase with increasing volume fraction (figure S.8). For particles with simple surface topographies this increase can be rescaled by a factor $C$ as determined in figure S.8. The reduced viscous and elastic stress amplitudes obtained are given by: $\Sigma_{v, r}=\frac{1}{C} \cdot \frac{G^{\prime \prime}-\eta_{\infty}^{\prime} \cdot 2 \pi f}{\eta_{\infty}^{\prime} \cdot 2 \pi f\left(\alpha^{*}=1\right)}$ and $\Sigma_{e, r}=\frac{1}{C} \cdot \frac{G^{\prime}}{\eta_{\infty}^{\prime} \cdot 2 \pi f\left(\alpha^{*}=1\right)}$.

Fig. 1 compares these reduced viscous and elastic stresses for the smooth silica-SM and the PMMA suspensions of different stabiliser thickness, all at $\phi_{\text {eff }} \approx 0.43$. To compare the steric and the electrostatic systems, we evaluate the curves at a reduced $\alpha^{*}>1$. Two limiting cases can be considered, i.e. the one of free draining and lubrication $[22,24,31]$. In the free draining limit, hydrodynamic interactions are effectively shielded and particles only interact at contact, leading to a 


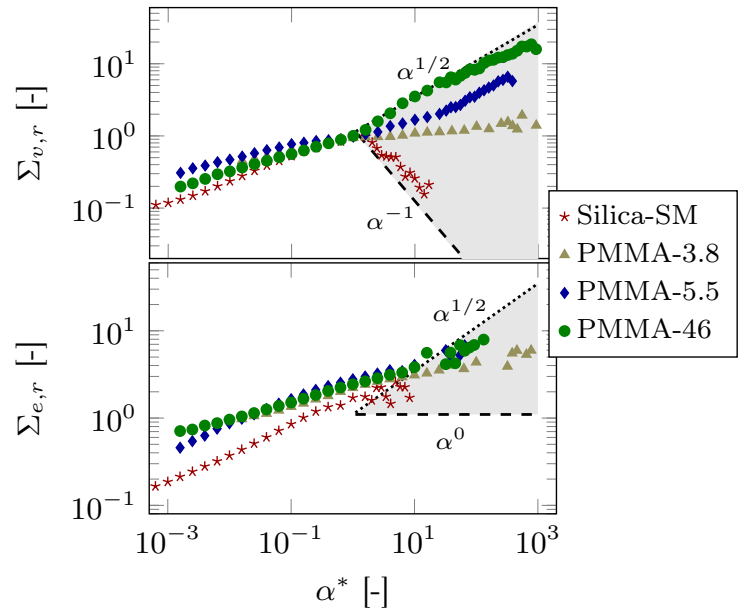

Figure 1: Reduced viscous (top) and elastic (bottom) stress amplitudes for particles with different surface characteristics at $\phi_{\text {eff }} \approx 0.43$. The lines present the theoretical limits for hydrodynamic lubrication (dashed) and repulsive hard-sphere (dotted) interactions. The dashed-dotted line denotes the limiting regimessiniting regimes aangeven welke het zijn

wat bedoel je met 'normal mode', moet hier bij de schaling ook geen referentie bij?

frequency response characterised by a normal mode response with both viscous and elastic stresses increasing as $\alpha^{1 / 2}$ (akin to the Rouse model). In the lubrication regime, with increasing frequency the thickness of the diffusive boundary layer decreases rapidly $\left(O\left(\alpha^{-1}\right)\right)$ and becomes smaller than the gap in the $\mathrm{HF}$ regime, resulting in a dissipative viscous component which dissapears $\Sigma_{v, r} \propto \alpha^{-1}$ and an elastic component which becomes constant $\Sigma_{e, r} \propto \alpha^{0}[22]$. One way to consider softer systems is that they affect the extent of shielding of the lubrication stresses $[24,32]$.

The frequency dependency of the viscous stress for the smooth silica-SM suspension displays a hydrodynamic lubrication scaling, consistent with some limited earlier observations [16] with the viscous extra stresses decreasing $\propto \alpha^{-1}$. The sterically stabilized PMMA suspensions show a remarkably different asymptotic behaviour depending on the thickness of the stabilisation layer. The PMMA-3.8 suspension, with a very thin stabilisation layer which is usually considered a model hard sphere system [33], does not show the response expected for hard spheres; the lubrication regime is not reached. For longer grafted chain lengths the exponent increases, and for the softest system the free draining limit appears to be reached. misschien aangeven wat het wil zeggen, maw, wat is de praktische consequentie?

Varying the volume fraction helps to further interrogate the interplay between the different contributions. For suspensions with "simple" surface topographies, such as the silica-SM suspensions in fig. 2 (a), the observed HF behaviour of the reduced stresses versus $\alpha_{p, B}$ is found to be independent of volume fraction and a pairwise potential approach is warranted. Similar behaviour was observed for the short sterically stabilised particles. However, for more complex topographies the situation changes. Fig. 2 (b) compares the reduced stress for PMMA-46 suspensions at different $\phi_{\text {eff }}$ as a function of frequency. The hydrodynamic dissipation inside the polymer brush is likely to be a complex function of the distance between the particles. For constant brush geometry, the hydrodynamic shielding can be determined [23, 36, 37] and has been used to calculate a high frequency moduli $[19,38]$. However, both the frequency and the volume fraction dependency of in particular the viscous stresses are insightful. Results for the PMMA-46 do not show a volume fraction independent behaviour: the scaling exponent of $\Sigma_{v, r}$ versus frequency decreases with particle concentration and may reflect brush compressibility. A reduced permeability inside the brush indeed entails a decrease in exponent [37]. At very high frequencies, the viscoelastic response of the brush itself might come into play. However, the $1^{\text {st }}$ Rouse relaxation mode of a 46PHSA strand in free suspension approximately occurs at $\sim 50 \mathrm{kHz}$, which is above the frequency window considered here $[39,40]$.

For suspensions of raspberry-like silica particles (fig. 2 (c),(d)) the behaviour is also observed to depend on volume fraction. There exists a concentration dependent region at intermediate frequencies $\left(\alpha_{p, B} \sim 10^{4}\right)$ for which the reduced viscous stresses strongly increase. This region is more pronounced for the rougher RO.5 compared to RO.4. The intermediate frequency regime can be viewed as a region where flow inside the rough layer dominates. As the roughness increases the magnitude of $\Sigma_{v, r}$ increases by about on order of magnitude. As the volume fraction is increased 


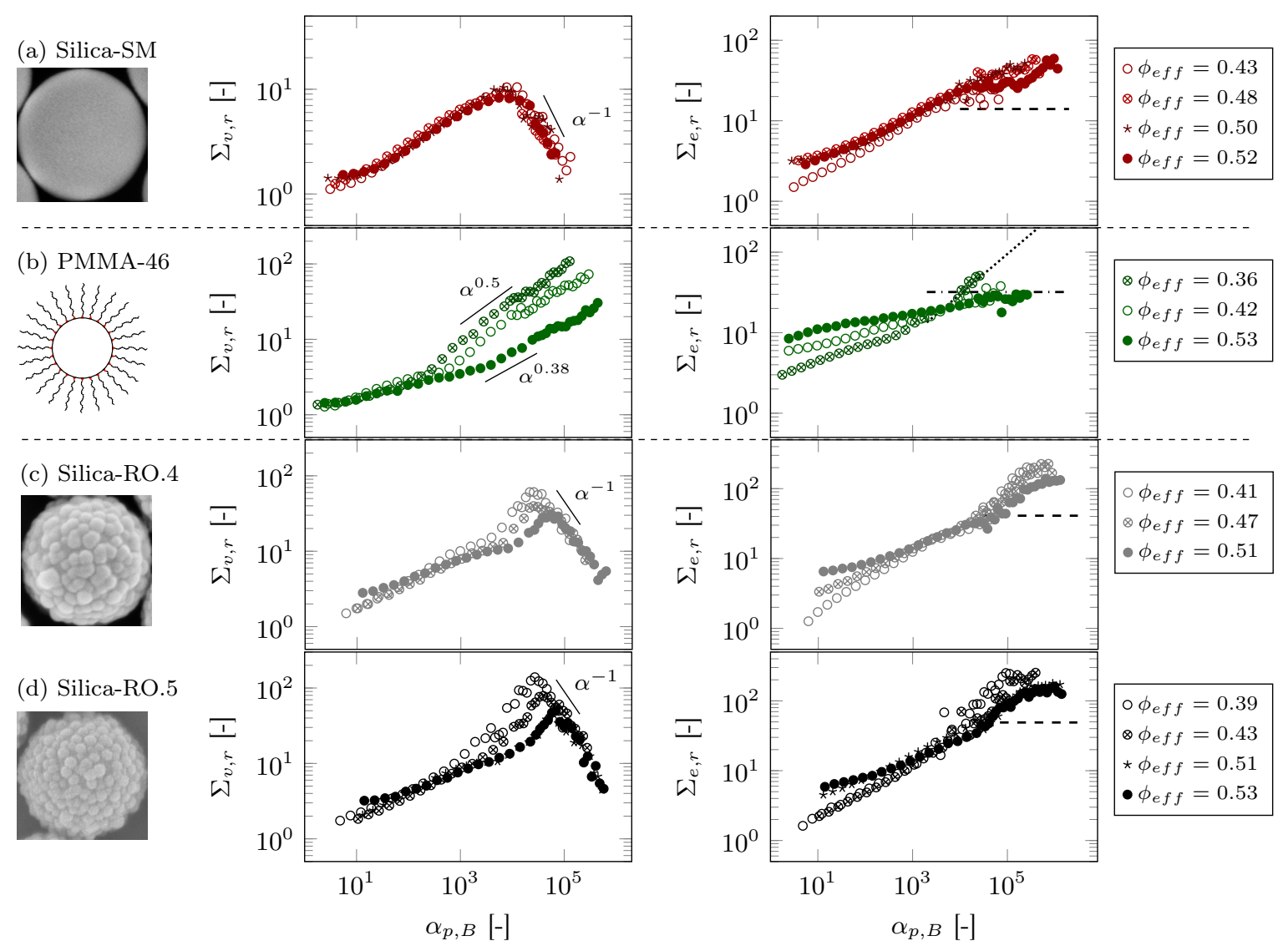

Figure 2: Reduced viscous (left) and elastic (right) stresses as a function of rescaled frequency for different volume fractions of smooth particles (a), particles with long polymeric brushes (b) and rough particles (c,d). (left) Solid lines indicate the scaling exponents. (right) Dashed, dotted and dashed-dotted lines present model predictions for $\Sigma_{e, r}$ based on lubrication [23], hard-sphere [31] and polymer-polymer interactions [34,35], respectively (suppl. mat. S6).

the peak shifts to higher frequencies and its magnitude is reduced. Most likely, as interparticle distances decrease further, the extent of this "porous" layer becomes smaller and dissipation is reduced. Detailed calculations of this localised hydrodynamic dissipation lie beyond the scope of the present work, but an interesting question for future research is that the inverse problem may be used to characterise the roughness of particles. At high frequencies $\left(\alpha_{p, B} \sim 10^{5}\right)$ the reduced viscous stresses strongly decrease again as a transition to a lubrication regime is observed. The frequency at which this occurs, shifts to higher values compared to the smooth silica-SM suspensions and is higher for increased roughness. This regime is observed when the thickness of the lubrication layer becomes on the same order as the distance between the asperities.

The HF limiting elastic stress is easier to rationalise, as variations of it are directly related to the interparticle potential. Model predictions were determined by taking into account the suppression of relaxation mechanisms which give rise to an elastic response by either lubrication stresses [23, 31], hard-sphere repulsions $[31,41]$ or repulsive interactions between polymer brushes [34,35,42] (suppl. mat. S6). The different limiting behaviours are given in fig. 2. The magnitude of the predicted HF elastic stress contributions, when rescaled in 
the same manner as the experimental data, qualitatively agree with measurements for the different suspensions. For the silica suspensions, plateau values based on the lubrication approximation [23] (suppl. mat. S6) underestimate the measured values, most likely due to electrostatic forces. For the polymeric brushes the elastic response shifts from the scaling expected for hard-sphere contacts [31] to the high frequency modulus given by a compressed polymer brush $[34,35,38]$. For the rough particles, as the lubrication regime is reached, the elastic stress also reaches a plateau. The magnitude of $\Sigma_{e, r}$ is higher than that expected for smooth spheres. A normal mode scaling as would be seen upon hard contacts is not observed under these equilibrium conditions.

The findings about the HF behaviour at nearequilibrium conditions can be compared to the behaviour of the suspensions under non-linear conditions [43]. As a specific application, the shear thickening behaviour of the different suspensions is considered. At such high stress levels, the confined suspensions undergo considerable normal stresses as well [2]. Fig. S.9 illustrates the behaviour of the different PMMA suspensions at $\phi_{\text {eff }} \approx 0.43$. The dispersions show a shear thickening state governed by lubrication stresses [44-48]. The viscosity increase is less pronounced for the PMMA-46 suspension: by effectively shielding the lubrication interactions between colloidal particles, the extent of shear thickening can hence be reduced [49]. The silica suspensions were studied at two distinct concentrations $\left(\phi_{\text {eff }} \approx 0.42-0.52\right.$; fig. S.10). The effective hydrodynamic volume fraction was able to capture the transition between a continuous and discontinuous shear thickening state for smooth and rough suspensions [9]. However, a difference in the stress mechanism can be seen depending on the surface topography. For smooth particles, the lubrication stresses diverging near contact are responsible for the arrest of motion and the subsequent viscosity increase. However, we postulate that the presence of asperities causes interlocking at sufficiently high volume fractions. This may bring about strong tangential interactions, which reduce the onset stress for shear thickening and shift the average pair probabilities of particles $[13,21]$. Whether this is due to hydrodynamic or truly frictional effects remains to be confirmed.
Concluding, the behaviour of both the viscous and elastic stress components at high frequency revealed an intricate interplay between surface topography, hydrodynamic lubrication stresses and interparticle interactions. The results on very soft or rough particles show that the changes in the hydrodynamic stresses with particle topology become a function of the volume fraction. For rough particles there is an increase of the dissipation through the hydrodynamic stresses, which decreases with increasing volume fraction.

\section{References}

[1] W. B. Russel, D. A. Saville, and W. R. Schowalter. Colloidal Dispersions. Cambridge University Press, 1989.

[2] J. Mewis and N. Wagner. Colloidal Suspension Rheology. Cambridge University Press, 2012.

[3] N. J. Wagner and J. F. Brady. Shear thickening in colloidal dispersions. Phys. Today, 62:27-32, 2009.

[4] M. M. Denn, J. F. Morris, and D. Bonn. Shear thickening in concentrated suspensions of smooth spheres in newtonian suspending fluids. Soft Matter, 14:170-184, 2018.

[5] D. Lootens, H. van Damme, Y. Hémar, and P. Hébraud. Dilatant flow of concentrated suspensions of rough particles. Phys. Rev. Lett., 95:268302, 2005.

[6] M. Wyart and M. E. Cates. Discontinuous shear thickening without inertia in dense non-brownian suspensions. Phys. Rev. Lett., 112:098302, 2014.

[7] R. Mari, R. Seto, J. F. Morris, and M. M. Denn. Discontinuous shear thickening in brownian suspensions by dynamic simulation. Proc. Natl. Acad. Sci. U.S.A., 112:15326-15330, 2015.

[8] N. Y. C. Lin, B. M. Guy, M. Hermes, C. Ness, J. Sun, W. C. K. Poon, and I. Cohen. Hydrodynamic and contact contributions to continuous shear thickening in colloidal suspensions. Phys. Rev. Lett., 115:228304, 2015. 
[9] J. R. Royer, D. L. Blair, and S. D. Hudson Rheological signature of frictional interactions in shear thickening suspensions. Phys. Rev. Lett., 116:188301, 2016.

[10] B. A. de L. Costello, P. F. Luckham, and Th. F. Tadros. Investigation of the interaction forces of polymer-coated surfaces using force balance, rheology, and osmotic pressure results. Langmuir, 8:464-468, 1992.

[11] S. J. O'Shea, M. E. Welland, and T. Rayment. An atomic force microscope study of grafted polymers on mica. Langmuir, 9:1826-1835, 1993.

[12] G. Bryant, S. R. Williams, L. Qian, I. K Snook, E. Perez, and F. Pincet. How hard is a colloidal "hard-sphere" interaction? Phys. Rev. E, 66:060501, 2002.

[13] C.-P. Hsu, S. N. Ramakrishna, M. Zanini, N. D. Spencer, and L. Isa. Roughnessdependent tribology effects on discontinuous shear thickening. Proc. Natl. Acad. Sci. U.S.A., 115:5117-5122, 2018.

[14] J. W. Goodwin, R. H. Ottewill, and A. Parentich. Compression studies on aqueous polystyrene latices. Colloid Polym. Sci., 268:1131-1140, 1990

[15] J. C. van der Werff, C. G. de Kruif, C. Blom, and J. Mellema. Linear viscoelastic behavior of dense hard-sphere dispersions. Phys. Rev. A, 39:795-807, 1989.

[16] T. Shikata and D. S. Pearson. Viscoelastic behavior of concentrated spherical suspensions. J. Rheol., 38:601-616, 1994.

[17] A. T. J. M. Woutersen, J. Mellema, C. Blom, and C. G. de Kruif. Linear viscoelasticity in dispersions of adhesive hard spheres. J. Chem. Phys., 101:542-553, 1994.

[18] J. Bergenholtz, N. Willenbacher, N. J. Wagner, B. Morrison, D. van den Ende, and J. Mellema. Colloidal charge determination in concentrated liquid dispersions using torsional resonance oscillation. J. Colloid Interface Sci., 202:430-440, 1998.
[19] P. A. Nommensen, M. H. G. Duits, D. van den Ende, and J. Mellema. Elastic modulus at high frequency of polymerically stabilized suspensions. Langmuir, 16:1902-1909, 2000.

[20] R. Seto, R. Mari, J. F. Morris, and M. M. Denn. Discontinuous shear thickening of frictional hard-sphere suspensions. Phys. Rev. Lett., 111:218301, 2013.

[21] L. C. Hsiao, S. Jamali, E. Glynos, P. F. Green, R. G. Larson, and M. J. Solomon. Rheological state diagrams for rough colloids in shear flow. Phys. Rev. Lett., 119:158001, 2017.

[22] J. F. Brady. The rheological behavior of concentrated colloidal dispersions. J. Chem. Phys., 99:567-581, 1993.

[23] R. A. Lionberger and W. B. Russel. Microscopic theories of the rheology of stable colloidal dispersions. Adv. Chem. Phys., 111:399$474,2000$.

[24] J. W. Swan, E. M. Furst, and N. J. Wagner. The medium amplitude oscillatory shear of semi-dilute colloidal dispersions. part i: Linear response and normal stress differences. $J$. Rheol., 58:307-337, 2014.

[25] J. Bergenholtz, F.M. Horn, W. Richtering, N. Willenbacher, and N. J. Wagner. Relationship between short-time self-diffusion and high-frequency viscosity in charge-stabilized dispersions. Phys. Rev. E, 58:R4088-R4091, 1998

[26] B. Schroyen, J. W. Swan, P. Van Puyvelde, and J. Vermant. Quantifying the dispersion quality of partially aggregated colloidal dispersions by high frequency rheology. Soft Matter, 13:7897-7906, 2017.

[27] L. Palangetic, K. Feldman, R. Schaller, R. Kalt, W. R. Caseri, and J. Vermant. From near hard spheres to colloidal surfboards. Faraday Discuss., 191:325-349, 2016.

[28] M. Zanini, C.-P. Hsu, T. Magrini, E. Marini, and L. Isa. Fabrication of rough colloids by heteroaggregation. Colloids Surf., A, 532:116$124,2017$. 
[29] A. Sierou and J. F. Brady. Accelerated stokesian dynamics simulations. J. Fluid Mech., 448:115-146, 2001.

[30] Z. Cheng, J. Zhu, P. M. Chaikin, S.-E. Phan, and W. B. Russel. Nature of the divergence in low shear viscosity of colloidal hard-sphere dispersions. Phys. Rev. E, 65:041405, 2002.

[31] R. A. Lionberger and W. B. Russel. High frequency modulus of hard sphere colloids. J. Rheol., 38:1885-1908, 1994.

[32] Z. Varga and J. W. Swan. Linear viscoelasticity of attractive colloidal dispersions. $J$. Rheol., 59:1271-1298, 2015.

[33] P. N. Pusey and W. van Megen. Phase behaviour of concentrated suspensions of nearly hard colloidal spheres. Nature, 320:340-342, 1986 .

[34] R. Zwanzig and R. D. Mountain. Highfrequency moduli of simple fluids. J. Chem. Phys., 43:4464, 1965.

[35] S. T. Milner, T. A. Witten, and M. E. Cates. Theory of the grafted polymer brush. Macromolecules, 21:2610-2619, 1988.

[36] A. A. Potanin and W. B. Russel. Hydrodynamic interaction of particles with grafted polymer brushes and applications to rheology of colloidal dispersions. Phys. Rev. E, 52:730$737,1995$.

[37] S. L. Elliott and W. B. Russel. High frequency shear modulus of polymerically stabilized colloids. J. Rheol., 42:361-378, 1998.

[38] M. H. G. Duits, P. A. Nommensen, D. van den Ende, and J. Mellema. High frequency elastic modulus of hairy particle dispersions in relation to their microstructure. Colloids Surf., A, 183-185:335-346, 2001.

[39] P. E. Rouse. A theory of the linear viscoelastic properties of dilute solutions of coiling polymers. J. Chem. Phys., 21:1272, 1953.

[40] B. H. Zimm. Dynamics of polymer molecules in dilute solution: Viscoelasticity, flow birefringence and dielectric loss. J. Chem. Phys., 24:269, 1956.
[41] N. F. Carnahan and K. E. Starling. Equation of state for noninteracting rigid spheres. $J$. Chem. Phys., 51:635, 1969.

[42] B. J. Maranzano and N. J. Wagner. Thermodynamic properties and rheology of sterically stabilized colloidal dispersions. Rheol. Acta, 39:483-494, 2000.

[43] J. F. Brady and J. F. Morris. Microstructure of strongly sheared suspensions and its impact on rheology and diffusion. J. Fluid Mech., 348:103-139, 1997.

[44] T. N. Phung, J. F. Brady, and G. Bossis. Stokesian dynamics simulation of brownian suspensions. J. Fluid Mech., 313:181-207, 1996.

[45] J. Mewis and G. Biebaut. Shear thickening in steady and superposition flows effect of particle interaction forces. J. Rheol., 45:799-813, 2001.

[46] V. Gopalakrishnan and C. F. Zukoski. Effect of attractions on shear thickening in dense suspensions. J. Rheol., 48:1321-1344, 2004.

[47] J. Bender and N. J. Wagner. Reversible shear thickening in monodisperse and bidisperse colloidal dispersions. J. Rheol., 40:899-916, 1996.

[48] J. Bergenholtz, J., J. F. Brady, J. F., and M. Vicic. The non-newtonian rheology of dilute colloidal suspensions. J. Fluid Mech., 456:239-275, 2002.

[49] L.-N. Krishnamurthy, N. J. Wagner, and J. Mewis. Shear thickening in polymer stabilized colloidal dispersions. J. Rheol., 49:13471360, 2005. 